\title{
Nondestructive high-throughput screening of nanopore geometry in porous membranes by imbibition
}

Cite as: Appl. Phys. Lett. 115, 113701 (2019); https://doi.org/10.1063/1.5119338

Submitted: 11 July 2019 . Accepted: 27 August 2019. Published Online: 10 September 2019

Luisa C. Cencha (D), Patrick Huber (D), Michael Kappl (D), George Floudas (D), Martin Steinhart (D), Claudio L.

A. Berli, and Raul Urteaga (D)
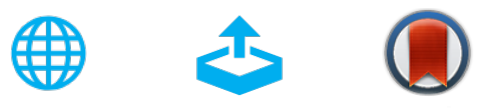

View Online

Export Citation

CrossMark

\section{ARTICLES YOU MAY BE INTERESTED IN}

Cation dependent electroosmotic flow in glass nanopores

Applied Physics Letters 115, 113702 (2019); https://doi.org/10.1063/1.5116760

Enhancement-mode $n-G a N$ gate $p$-channel heterostructure field effect transistors based on GaN/AIGaN 2D hole gas

Applied Physics Letters 115, 112103 (2019); https://doi.org/10.1063/1.5119985

Two-terminal terahertz detectors based on AlGaN/GaN high-electron-mobility transistors

Applied Physics Letters 115, 111101 (2019); https://doi.org/10.1063/1.5114682

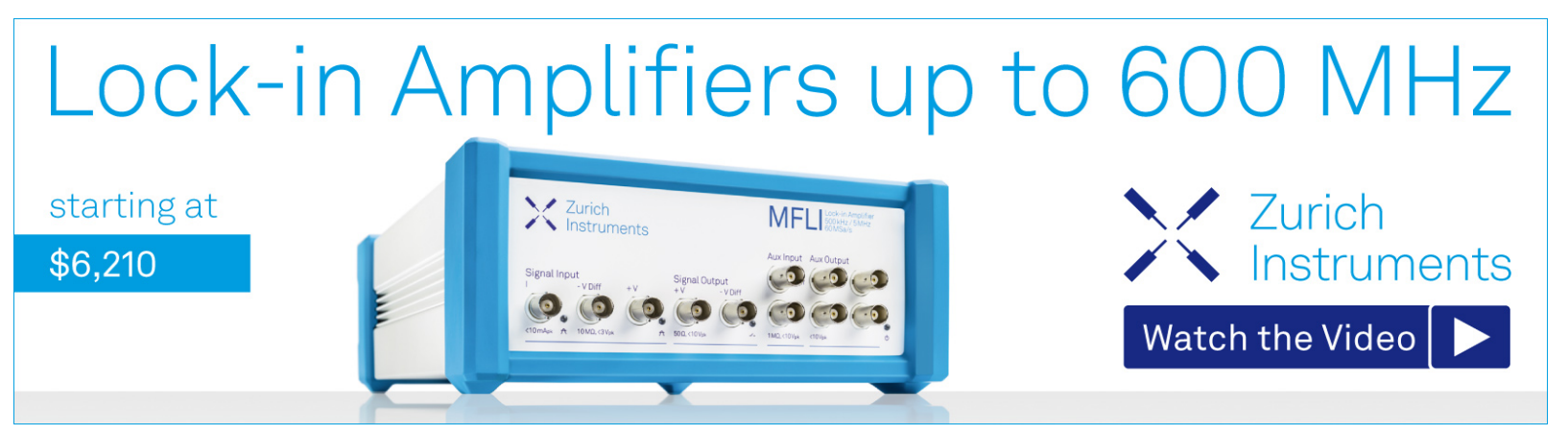




\title{
Nondestructive high-throughput screening of nanopore geometry in porous membranes by imbibition
}

Cite as: Appl. Phys. Lett. 115, 113701 (2019); doi: 10.1063/1.5119338

Submitted: 11 July 2019 - Accepted: 27 August 2019 .

Published Online: 10 September 2019

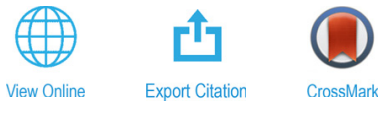

Luisa G. Cencha, ${ }^{1}$ iD Patrick Huber, ${ }^{2}$ (D Michael Kappl, ${ }^{3}$ (iD George Floudas, ${ }^{3,4}$ (iD Martin Steinhart, $^{5}$ Claudio L. A. Berli, ${ }^{6}$ and Raul Urteaga, a) iD

\author{
AFFILIATIONS \\ 'IFIS-Litoral (Universidad Nacional del Litoral-CONICET), Guemes 3450, 3000 Santa Fe, Argentina \\ ${ }^{2}$ Institute of Materials Physics and Technology, Hamburg University of Technology, 21073 Hamburg-Harburg, Germany \\ ${ }^{3}$ Max Planck Institute for Polymer Research, D-55128 Mainz, Germany \\ ${ }^{4}$ Department of Physics, University of Ioannina, P.O. Box 1186, 45110 loannina, Greece \\ ${ }^{5}$ Institut für Chemie neuer Materialien, Universität Osnabräck, Barbarastr. 7, 49076 Osnabrück, Germany \\ ${ }^{6}$ INTEC (Universidad Nacional del Litoral-CONICET), Predio CCT CONICET Santa Fe, RN 168, 3000 Santa Fe, Argentina

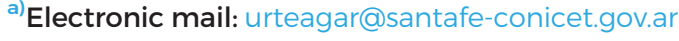

\begin{abstract}
A fluid dynamic model for imbibition into closed-end, axisymmetric pores having diameters that change as a function of the pore depth is presented. Despite the fact that liquid invasion into nonbranched closed-end pores is characterized by a wealth of different transient and/or metastable nonequilibrium stages related to precursor film formation, we show that a simple hydraulic model accounting for geometry- and air compression-induced deviations from classical Lucas-Washburn dynamics precisely describes the imbibition dynamics except at the late stage. The model was validated by laser interferometry experiments with submillisecond temporal resolution. Imbibition of three simple liquids (isopropanol, ethanol, and hexane) into self-ordered anodic alumina membranes containing arrays of parallel closed-end nanopores characterized by slight conicity was studied. The model provides an improved description of nanoscale fluid dynamics and allows geometric characterization of nanoporous membranes by their imbibition kinetics accounting for the back pressure of the compressed gas. Thus, a precise calibration of porous membranes with simple liquids becomes possible, and changes in the mean pore diameter as a function of the pore depth can be assessed.
\end{abstract}

Published under license by AIP Publishing. https://doi.org/10.1063/1.5119338

Nanoporous media, in particular, monolithic porous membranes, play an increasing scientific and technological role. ${ }^{1-3}$ Among the applications of porous membranes stand out filtration and separation processes, ${ }^{4-6}$ the study of confinement effects in soft matter, ${ }^{7-9}$ and energy harvesting. ${ }^{10,11}$ However, their characterization in terms of pore size and pore homogeneity is still an extremely demanding experimental task, even though it is of crucial importance for the interpretation of experiments and for the understanding of functional performance of nanoporous membranes. The sample requirements for the most prominent characterization methods of nanoporous materials, such as volumetric sorption isotherm measurements, mercury porosimetry, and electron microscopy, necessitate powders or small sample pieces and thus a destruction of the porous medium. Here, we present a theoretical analysis showing that simple, capillarity-driven filling experiments allow one to nondestructively analyze the pore geometry of macroscopic porous membranes with nanoscale spatial resolution in terms of pore diameter (gradients). Moreover, we validate this method by experiments on the imbibition dynamics of several selected simple liquids in archetypical monolithic nanoporous media, i.e., anodic aluminum oxide (AAO) membranes.

Self-ordered nanoporous $\mathrm{AAO}^{12,13}$ membranes containing arrays of straight parallel nanopores are easily accessible by an aluminum foil two-step anodization procedure introduced by Masuda and co-workers. ${ }^{12}$ This material has been used extensively as mold for the production of one-dimensional nanostructures and as the pore model for the study of structure formation as well as dynamic processes 
inside the confinement of nanopores. ${ }^{7,8,13-15}$ The pores of an asanodized self-ordered AAO membrane are closed at its bottoms, and the pore diameter is slightly larger at the top surface (pore mouths), ${ }_{16,17}$ with a typical variation of less than $1 \mathrm{~nm}$ per micrometer distance from the pore mouths. While this pore diameter variation is hard to detect with classical analytical methods, this geometric feature can substantially modify the dynamics of capillary imbibition even for a very small opening angle. ${ }^{18} \mathrm{~A}$ model to describe the imbibition dynamics of closed-end pores with diameter variations along the pores is not available at present, and this is one of the objectives of the present work.

There are previous studies on the influence of geometrical variations on the imbibition kinetics in single capillaries or in more complex porous media. For example, Reyssat et al., ${ }^{19,20}$ Erickson et al., ${ }^{21}$ and Liou et al. ${ }^{22}$ studied imbibition with changing hydraulic permeability as a function of imbibition advancement. No trapped gas (typical of deadend pores) has been considered in these studies. By contrast, Phan et al., ${ }^{23}$ Radiom et al., ${ }^{24}$ and Acquaroli et al. ${ }^{25}$ studied capillary filling in closed end cylindrical geometries considering trapped gas and thus with constant hydraulic permeability.

Later on, the inverse problem of capillary filling in nonuniform capillaries was studied, which consists in determining the axial variation of the capillary radius starting from the experimental data of the meniscus position as a function of time. ${ }^{18}$ In particular, for conical and open-end capillaries, the capillary imbibition of non-Newtonian inelastic fluids was also considered. ${ }^{26}$

This paper presents a model of capillary imbibition of Newtonian fluids into axisymmetric closed-end nanochannels that may exhibit channel diameter variations along the channel axes [Fig. 1(a)]. In combination with interferometric techniques, our model enables nondestructive screening of pore geometries, thus yielding the mean pore diameters as a function of the distance to the pore mouths. Our model considers the interplay of capillary imbibition and back pressure originating from the compression of air trapped in the pores away from the pore mouths. Assuming that the pores in a membrane are noninterconnected and that they have the

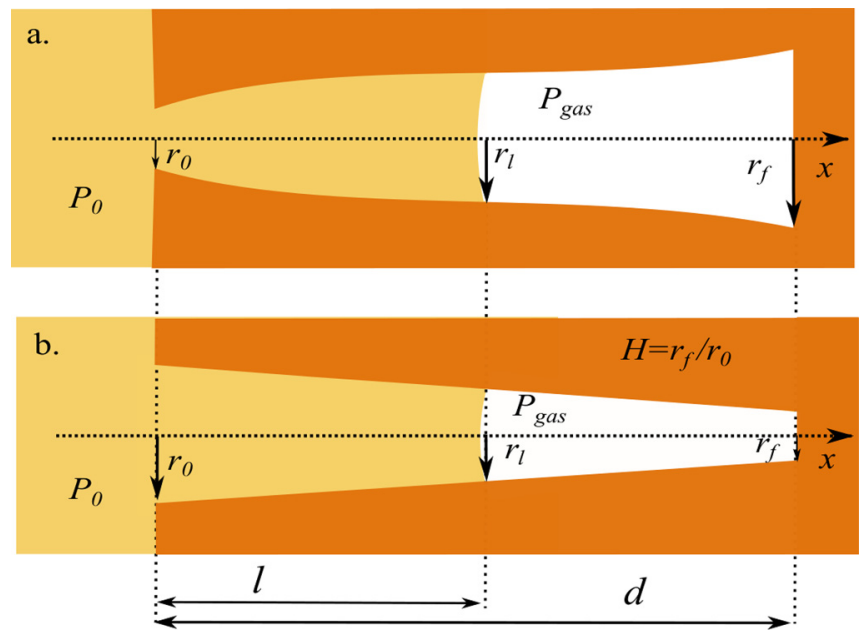

FIG. 1. Schematic representation of capillary imbibition in closed-end channels with (a) arbitrary variation in the channel diameter along the channel axis and (b) radius variation $r(x)=r_{0}+B x$. same shape and size, one may consider capillary imbibition in one of the pores as being representative of the fluid front evolution in the entire membrane. For nanoscale pores, inertial effects can be safely neglected for the time-scales studied here ${ }^{27}$ and a parabolic velocity profile is assumed (Hagen-Poiseuille flow). The implicit boundary condition is the presence of an infinite bulk reservoir of the invading liquid. It is also assumed that the gas in the channel has the initial pressure of the reservoir, $P_{0}$, and that it is isothermally compressed, while the liquid front advances. The model does neither take into account the gas dissolution in the fluid nor gas diffusion through the channel walls.

The driving force of the capillary imbibition is the pressure difference $\Delta P=P_{0}-\left(P_{\text {gas }}-P_{c}\right)$, where $P_{\text {gas }}$ is the pressure of the trapped gas and $P_{c}$ is the pressure difference arising across the meniscus as a consequence of the surface tension (Laplace's law), $P_{c}=2 \gamma \cos \theta / r_{l}$, where $\gamma$ is the surface tension of the invading liquid, $\theta$ is the contact angle between the fluid and the outline of the contact pore surface, and $r_{l}$ is the channel radius at position $l$. Assuming that the compression of the trapped gas is isothermal, the pressure rise in the trapped gas is proportional to the ratio between the initial volume $V_{0}$ (total volume of the channel) and the remaining gas volume $V_{l}$. Therefore, $P_{\text {gas }}=P_{0} V_{0} / V_{l}$ (Boyle's law), and the pressure difference is $\Delta P$ $=P_{c}-P_{0} \int_{0}^{l} r(x)^{2} d x / \int_{l}^{d} r(x)^{2} d x$. For a nonuniform axisymmetric channel, the relationship between the volume flow rate $q$ and the pressure jump at the advancing liquid meniscus is ${ }^{19} q=\Delta P / R_{h}$ $=\Delta P /\left(\frac{8 \eta}{\pi} \int_{0}^{l} r(x)^{-4} d x\right)$, where $R_{h}$ is the hydraulic resistance of the channel and $\eta$ is the fluid viscosity. By combining these equations and by considering that $q=\pi r_{l}^{2}(d l / d t)$, the following differential equation for the meniscus velocity is obtained:

$$
\frac{d l}{d t}=\frac{\frac{2 \gamma \cos \theta}{r_{l}}-P_{0} \frac{\int_{0}^{l} r(x)^{2} d x}{\int_{l}^{d} r(x)^{2} d x}}{8 \eta r(x)^{2} \int_{0}^{l} r(x)^{-4} d x} .
$$

The above equation is a general expression that describes the evolution of the capillary imbibition front in any arbitrary channel geometry $r(x)$ with the initial condition $l=0$ and $P=P_{0}$ for $t=0$. It is worth noting that for uniform $r(x)$ and open channels $\left(P_{0}=0\right)$, Eq. (1) becomes the classical Lucas-Washburn (LW) equation ${ }^{28,29}$ for capillary imbibition. Also, for uniform $r(x)$ and closed channels, Eq. (1) reproduces the results obtained by Acquaroli et al. ${ }^{25}$ for closed-end cylindrical capillaries. Finally, for arbitrary $r(x)$ and $P_{0}=0$, Eq. (1) reduces to the results obtained by Reyssat et al. ${ }^{19}$ for open channels.

The particular case of a truncated conical pore is shown in Fig. $1(\mathrm{~b})$, where $r(x)=r_{0}+B x=r_{0}+\frac{r_{f}-r_{0}}{d} x$. Here, $r_{0}$ and $r_{f}$ are the initial and final radii of the channel and $d$ is the membrane thickness. In this case, introducing the following dimensionless parameters $\bar{r} \equiv \frac{r_{l}}{r_{0}}, H \equiv r_{f} / r_{0}$, and $\alpha \equiv \frac{P_{0}}{2 \gamma \cos \theta / r_{0}}$, Eq. (1) can be written as

$$
\frac{d l}{d t}=\frac{3 B \gamma \cos \theta}{4 \eta}\left\{\frac{1-\alpha \bar{r}\left(\frac{\bar{r}^{3}-1}{H^{3}-\bar{r}^{3}}\right)}{\bar{r}^{3}-1}\right\} .
$$


This expression describes the fluid front velocity in closed-end conical pores at a given position $x=l$ that corresponds to the channel radius $r_{l}$. Note that Eq. (2) can be made explicit by using $\bar{r}=1$ $+(H-1) \frac{l}{d}$.

A vanishing meniscus movement and thus a final capillary filling position can be easily derived from Eq. (2) for the condition $\frac{d l}{d t}=0$, i.e.,

$$
\alpha \bar{r}\left(\frac{\bar{r}^{3}-1}{H^{3}-\bar{r}^{3}}\right)=1 .
$$

Figure 2 shows the reached normalized hydraulic equilibrium positions $\bar{l}=\frac{l}{d}$ for the $\bar{r}$ obtained by solving Eq. (3) for different values of $H$ and $\alpha$. It can be seen that, for small values of $\alpha$, the maximum position $\bar{l}$ is obtained for $H \approx 1$, i.e., for an almost cylindrical shape. This can be rationalized as a trade-off between achieving high capillary pressure (small pore radius) and having a large volume available to accommodate compressed gas (large pore radius). On the other hand, it is interesting to note that for values of $H \approx 1$, equilibrium position values are relatively constant and can be calculated simply from the expression obtained for cylinders, ${ }^{25}$ i.e., $\bar{l}=\frac{1}{1+\alpha}$.

Experimentally, the capillary imbibition dynamics of liquids in porous matrixes can be accurately measured by laser interferometry, a nondestructive method that measures the light reflected or transmitted by thin membranes during fluid imbibition.

By using Looyenga-Landau-Lifshitz mixing rules, ${ }^{17}$ the change in the normalized optical thickness $\Delta e_{o p}$ can be accurately estimated as the relative volume change of fluid inside the membrane $\bar{V}=V_{0 l} /$ $V_{0 d}$, where $V_{0 l}$ is the occupied volume by the fluid and $V_{0 d}=V_{0 l}$ $+V_{l d}$ is the total volume available to be filled. For the particular case of cylindrical pores, the porosity is uniform across the membrane thickness, and $\bar{V}$ equals the normalized front position $l / d=\bar{l}$. In the case of conical pores, $l(t)$ and $V_{0 l}(t)$ are related by the cone volume expression $V_{0 l}(t)=(\pi / 3) l(t)\left(r_{0}^{2}+r_{l}^{2}+r_{0} r_{l}\right)$.

The interferometric measurements can be directly related to the quantity of liquid infiltrated into the membrane as a function of time. In such a way, the measurement of the reflectance or transmittance as

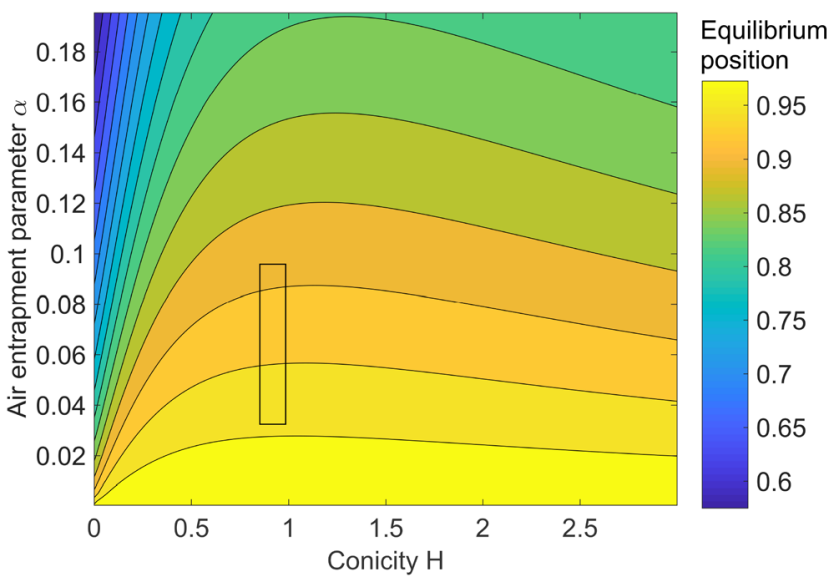

FIG. 2. Normalized hydraulic equilibrium position $\bar{T}$ in closed-end conical pores having different combinations of conical degree $(H)$ and trapped gas content $(\alpha)$. The box in the figure indicates the region that corresponds to values attained in our experiments. a function of time during imbibition can be transformed to the volume of infiltrated liquid as a function of imbibition time. The experimental setup for laser interferometry is described in the supplementary material, and more details on the method can be found in previous works. $^{16,17,25,30}$

As the pore model, we used self-ordered AAO with a nominal pore diameter much smaller than infrared wavelengths, and the selfordered AAO membranes themselves were transparent. Hence, they can be modeled as effective optical medium. ${ }^{31}$ The total number of oscillations in each experiment reveals the optical thickness variation and hence the average refractive index of the membrane. Then, the average membrane porosity can be calculated by using an effective medium model. ${ }^{16}$ We used hexane [Fig. 3(a)], isopropanol [Fig. 3(b)], and ethanol [Fig. 3(c)] as simple model fluids. The relevant properties of the model fluids are summarized in the supplementary material.

Self-ordered AAO membranes were prepared by a two-step anodization process following procedures reported by Masuda and co-workers. ${ }^{12,32}$ Free-standing self-ordered AAO membranes were
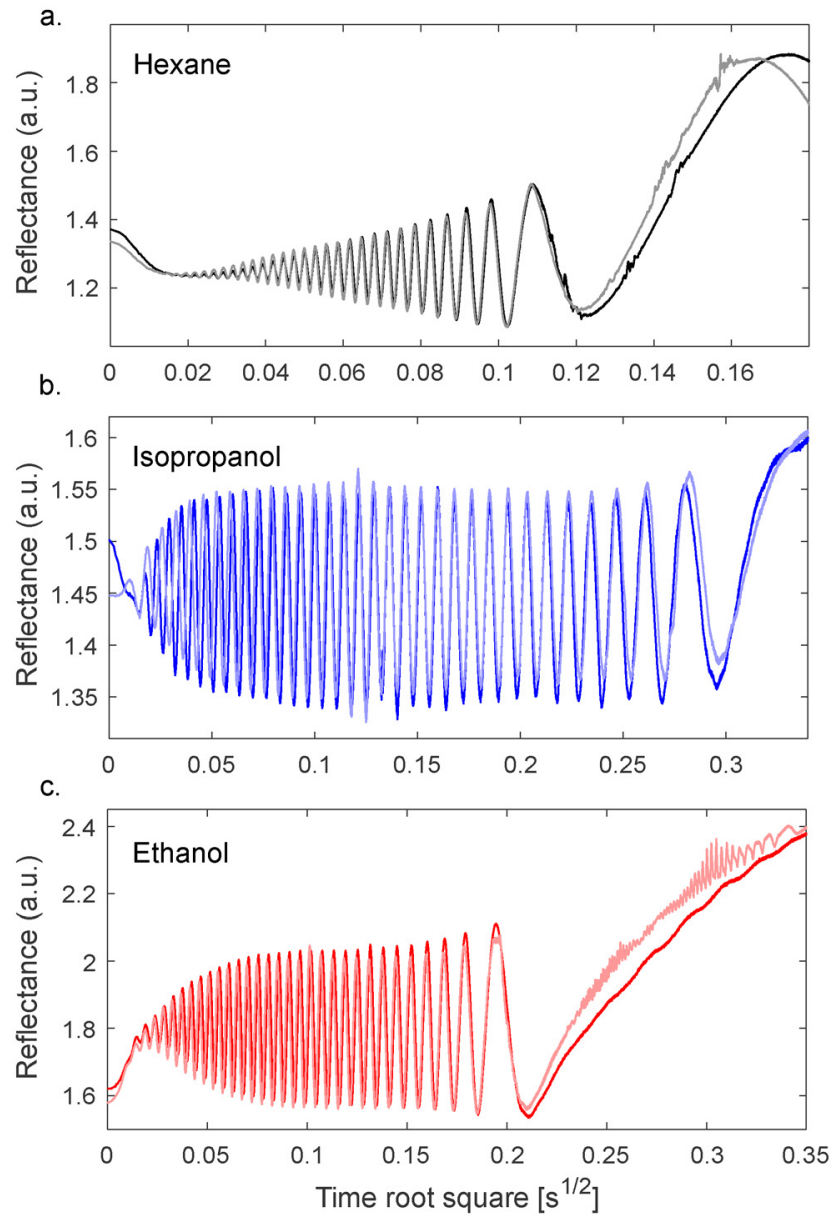

FIG. 3. Reflectance of self-ordered AAO with a nominal pore diameter of $65 \mathrm{~nm}$ and a pore depth of $108 \mu \mathrm{m}$ as a function of the square root of time during the imbibition with (a) hexane, (b) isopropanol, and (c) ethanol. Two representative measurements for each fluid are shown. 
obtained with pore bottoms closed by barrier oxide. Self-ordered AAO membranes with a nominal pore diameter of $65 \mathrm{~nm}$ and a pore depth of $\sim 108 \mu \mathrm{m}$, with a nominal pore diameter of $35 \mathrm{~nm}$ and a pore depth of $\sim 89 \mu \mathrm{m}$, and with a nominal pore diameter of $25 \mathrm{~nm}$ and a pore depth of $\sim 100 \mu \mathrm{m}$ were obtained. More details on the preparation process, as well as on pore characterization by SEM and FIB measurements, are presented in the supplementary material.

All measurements were carried out at exactly the same location, while the membrane was allowed to dry between the imbibition experiments. All measurements were repeated several times and showed excellent reproducibility. For the sake of clarity, only two measurements for each fluid are shown in Fig. 3. The average membrane porosities obtained from laser-interferometric measurements with ethanol, isopropanol, and hexane coincide and amount to $\sim 38 \%$. The theoretical porosity value for a perfect hexagonal pore lattice with a lattice constant of $105 \mathrm{~nm}$ and a constant pore diameter of $65 \mathrm{~nm}$ amounts to $35 \%$.

We derived the dependence of the relative volume change of fluid inside the membrane $\bar{V}$ on the elapsed imbibition time theoretically from the data displayed in Fig. 3 following methods described elsewhere. $^{16,17,25}$ The experimental volume change $\bar{V}$ was normalized with its final value $\left(\bar{V}_{\max }\right)$ and fitted with the fluid dynamic model of Eq. (2) together with the relationship between $l(t)$ and $V(t)$, which is the cone volume expression. The results are shown in Fig. 4, where only two free parameters, $H=0.88$ and $r_{0}$ with $2 r_{0}=74 \mathrm{~nm}$ (and $2 r_{f}$ $=65 \mathrm{~nm}$ ), were used to fit all datasets of all fluids with excellent reproduction of the experimental results.

From the SEM cross-sectional images, it was found that mean top and bottom diameter $2 r_{0}=66 \mathrm{~nm}$ and $2 r_{f}=57 \mathrm{~nm}$, respectively, and from the FIB analysis, it was found that $2 r_{0}=62 \mathrm{~nm}$ and $2 r_{f}$ $=51 \mathrm{~nm}$ (see the supplementary material). Therefore, the values obtained from the imbibition are in reasonable agreement with the

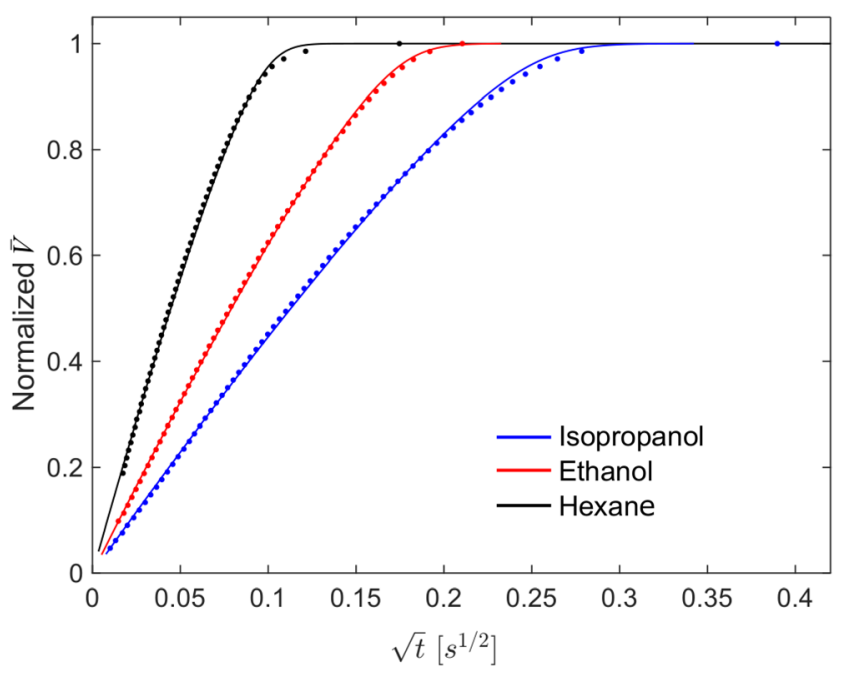

FIG. 4. Normalized relative volume change of fluid inside the membrane $\bar{V}$ experimentally obtained by laser interferometry as a function of the elapsed imbibition time $\sqrt{t}$ for the imbibition of self-ordered AAO with a nominal pore diameter of $65 \mathrm{~nm}$ and a pore depth of $108 \mu \mathrm{m}$ with ethanol, isopropanol, and hexane. Solid lines correspond to the prediction of the fluid dynamic model [Eq. (2)]. Fitting parameters were $H=0.88$ and $2 r_{0}=74 \mathrm{~nm}\left(2 r_{f}=65 \mathrm{~nm}\right)$. microscopy analysis. It is worth mentioning that the characterization of absolute lengths and distances in the sub-100-nm range by SEM is usually associated with significant errors of $10 \%-15 \%$ or more. ${ }^{33}$ Even if the instrumental error is minimum, the measured lengths will depend strongly on the gray threshold value chosen to delimit the edges of the object is being measured. ${ }^{33}$ The measured slight decrease in the AAO pore diameter is consistent with the fact that the pore mouths are longer exposed to the electrolyte solution during anodization. The conical shape of the pores was also observed by the analysis of SEM and FIB images, which yielded $H=0.86$ and $H=0.82$, respectively, showing excellent agreement with the imbibition results. Regarding the uncertainty associated with the estimation of $H$, the influence of the image measurement error is less important, since the same gray threshold is used for the top and bottom images. ${ }^{33}$ Interferometric imbibition experiments with self-ordered AAO having pore diameters of $35 \mathrm{~nm}$ and $25 \mathrm{~nm}$ and isopropanol yielded $H$ values of 0.92 and 0.84 , respectively.

The question arises as to how the simple hydraulic imbibition model elaborated above is compatible with the rich phenomenology of transient and metastable imbibition morphologies related to the formation of precursor films ${ }^{34-37}$ on the pore walls. Since the pore walls of self-ordered AAO are high-energy surfaces terminated by polar hydroxyl groups, the model fluids used here will spread on the pore walls driven by strong interfacial interactions. Thus, if an AAO pore of finite size is in contact with an "infinite" reservoir of an invading liquid having higher affinity to the AAO pore walls than air, in the thermodynamic equilibrium, the AAO pores will correspond to a state in which the interfacial energy is minimized-i.e., the complete filling of the AAO pores with the invading liquid, once the gas has completely diffused into the liquid. Actually, immediately after the hydraulic equilibrium is reached, a slow process of gas dissolution yields a complete filling of the pores finally reaching the thermodynamic equilibrium, as was verified in similar systems. ${ }^{23}$ Laser interferometry also has the potential to study gas dissolution in the fluid at later imbibition stages. Preliminary experiments suggested that after the hydraulic equilibrium is reached, air diffusion through the liquid eventually leads to complete filling of the pore volume with the invading liquid. This last stage can provide relevant information about the fluid diffusivity under strong confinement; however, it is not necessary for the membrane characterization here proposed.

The imbibition dynamics of simple Newtonian liquids invading nonconnected close-end pores with diameters that vary as a function of the distance from the pore mouth were analyzed by a theoretical model. The liquids investigated spread on the pore walls of self-ordered AAO used as the pore model. An unchanged no-slip velocity boundary condition on the pore walls and no changes in the fluidity and capillarity compared to the bulk systems are expected. ${ }^{7,38}$ Moreover, potential immobile adsorption monolayers at the pore walls ${ }^{37,39-41}$ affect the fluid dynamics for the comparably large pore sizes only marginally. These retained macroscopic hydrodynamics allowed us to infer the implications of the pore morphology and of air-entrapment at dead ends of pores on the calculation of viscosities $\eta$ from capillary filling experiments. Two effects were considered: trapped air and pore conicity. The former creates a curvature at long times in the classical LW equation, whereas the latter underestimates the fluid viscosity. Neglecting the conicity of the pores and the air entrapment effect can result in errors of up to $30 \%$ in the determination of the fluid viscosity. 
On the other hand, the correct measurement of the membrane thickness is essential since this value quadratically affects the magnitude of the obtained viscosity.

An existing limitation of AAO membranes is the significant variability between different samples of the same nominal pore size. This effect introduces a considerably dispersion in the measurements. To overcome this limitation, a calibration measurement with simple liquids, as exemplified here, would allow us to strongly reduce the error in viscosity calculations, by using precise correction factors.

The proposed methodology is able to accurately identify the internal geometry of nanochannels in a nondestructive manner, by using a simple experimental setup. These are clear advantages in relation to other methods routinely employed, like sorption isotherms, electron microscopy, and cryoporometry, and thus, the method can be of particular interest for the characterization of nanoporous matrices.

Our study provides a sound base for future optofluidic studies on complex liquids, in particular, polymeric and liquid-crystalline systems. For these fluids, deviations from the classical LW behavior can result from intrinsic fluid properties, confinement-induced fluid-wall interactions, most prominently shear-rate dependent viscosities, ${ }^{42}$ slippage effects, and immobile boundary layers due to absorption at the pore walls. $^{7-9,27,37,40}$ Therefore, a sound discrimination with respect to geometry- and air-entrapment-induced deformation is of crucial importance.

See the supplementary material for a detailed description of the experimental setup and additional data on fabrication and characterization of AAO membranes.

The authors thank the German Research Foundation for funding (PAK 949 "Nanostructured Glasses and Ceramics," Nos. Hu850/9-1, STE1127/19-1, and Projektnummer 192346071-SFB 986) as well as C. Hess and C. Schulz-Kölbel for technical support. L. G. Cencha thanks the Bunge and Born and Williams Foundations, which in collaboration with the Max Planck Society partially supported this work.

\section{REFERENCES}

${ }^{1}$ P. Guo, J. Huang, Y. Zhao, C. R. Martin, R. N. Zare, and M. A. Moses, Small 14, 1703493 (2018).

${ }^{2}$ Q. Wang and R. Chen, Nano Lett. 18, 3096 (2018).

${ }^{3}$ J. Buchheim, K.-P. Schlichting, R. M. Wyss, and H. G. Park, ACS Nano 13, 134 (2019).

${ }^{4}$ H. Wang, X. Hu, Z. Ke, C. Z. Du, L. Zheng, C. Wang, and Z. Yuan, Nanoscale Res. Lett. 13, 284 (2018).

${ }^{5}$ M. S. S. A. Saraswathi, A. Nagendran, and D. Rana, J. Mater. Chem. A 7, 8723 (2019).

${ }^{6}$ Z. Wang, A. Wu, L. C. Ciacchi, and G. Wei, Nanomaterials 8, 65 (2018).

${ }^{7}$ P. Huber, J. Phys.: Condens. Matter 27, 103102 (2015).
${ }^{8}$ Y. Yao, Y. Suzuki, J. Seiwert, M. Steinhart, H. Frey, H.-J. Butt, and G. Floudas, Macromolecules 50, 8755 (2017).

${ }^{9}$ Y. Yao, S. Alexandris, F. Henrich, G. Auernhammer, M. Steinhart, H.-J. Butt, and G. Floudas, J. Chem. Phys. 146, 203320 (2017).

${ }^{10}$ X. Zhu, J. Hao, B. Bao, Y. Zhou, H. Zhang, J. Pang, Z. Jiang, and L. Jiang, Sci. Adv. 4, eaau1665 (2018).

${ }^{11}$ A. Siria, M.-L. Bocquet, and L. Bocquet, Nat. Rev. Chem. 1, 0091 (2017).

${ }^{12}$ H. Masuda and K. Fukuda, Science 268, 1466 (1995).

${ }^{13}$ W. Lee and S.-J. Park, Chem. Rev. 114, 7487 (2014).

${ }^{14} \mathrm{M}$. Steinhart, in Self-Assembled Nanomaterials II: Nanotubes, Advances in Polymer Science Vol. 220 (Springer International Publishing, 2008).

${ }^{15}$ J. Martin, J. Maiz, J. Sacristan, and C. Mijangos, Polymer 53, 1149 (2012).

${ }^{16}$ R. Urteaga, L. N. Acquaroli, R. R. Koropecki, A. Santos, M. Alba, J. Pallarés, L. F. Marsal, and C. L. A. Berli, Langmuir 29, 2784 (2013).

${ }^{17}$ R. Urteaga and C. L. A. Berli, "Nanoporous anodic alumina for optofluidic applications," in Nanoporous Alumina (Springer International Publishing, 2015).

${ }^{18}$ E. Elizalde, R. Urteaga, R. R. Koropecki, and C. L. Berli, Phys. Rev. Lett. 112, 134502 (2014).

${ }^{19}$ M. Reyssat, L. Courbin, E. Reyssat, and H. A. Stone, J. Fluid Mech. 615, 335 (2008).

${ }^{20}$ M. Reyssat, L. Y. Sangne, E. A. van Nierop, and H. A. Stone, Europhys. Lett. 86, 56002 (2009).

${ }^{21}$ D. Erickson, D. Li, and C. Park, J. Colloid Interface Sci. 250, 422 (2002).

${ }^{22}$ W. W. Liou, Y. Peng, and P. E. Parker, J. Colloid Interface Sci. 333, 389 (2009).

${ }^{23}$ V. N. Phan, N.-T. Nguyen, C. Yang, P. Joseph, L. Djeghlaf, D. Bourrier, and A.M. Gue, Langmuir 26, 13251 (2010).

${ }^{24}$ M. Radiom, W. K. Chan, and C. Yang, Microfluid. Nanofluid. 9, 65 (2010).

${ }^{25}$ L. N. Acquaroli, R. Urteaga, C. L. A. Berli, and R. R. Koropecki, Langmuir 27(5), 2067 (2011).

${ }^{26}$ C. L. A. Berli and R. Urteaga, Microfluid. Nanofluid. 17(6), 1079 (2014).

${ }^{27}$ S. Gruener, H. E. Hermes, B. Schillinger, S. U. Egelhaaf, and P. Huber, Colloid. Surf. A 496, 13-27 (2016).

${ }^{28}$ R. Lucas, Kolloid-Z. 23, 15 (1918).

${ }^{29}$ E. W. Washburn, Phys. Rev. 17, 273 (1921).

${ }^{30}$ C. Eckstein, E. Xifré-Pérez, M. P. i Batalla, J. Ferré-Borrull, and L. F. Marsal, Langmuir 32, 10467 (2016).

${ }^{31}$ L. Landau, J. Bell, M. Kearsley, L. Pitaevskii, E. Lifshitz, and J. J. Sykes, Electrodynamics of Continuous Media, Course of Theoretical Physics (Elsevier Science, 2013).

${ }^{32}$ H. Masuda, F. Hasegwa, and S. Ono, J. Electrochem. Soc. 144, L127 (1997).

${ }^{33}$ M. T. Postek and A. E. Vladár, Scanning 35, 355 (2013).

${ }^{34}$ D. Ausserré, A. M. Picard, and L. Léger, Phys. Rev. Lett. 57, 2671 (1986).

${ }^{35}$ L. Léger, M. Erman, A. M. Guinet-Picard, D. Ausserré, and C. Strazielle, Phys. Rev. Lett. 60, 2390 (1988).

${ }^{36}$ L. Léger and J. F. Joanny, Rep. Prog. Phys. 55, 431 (1992).

${ }^{37}$ S. Gruener and P. Huber, Phys. Rev. Lett. 103, 174501 (2009).

${ }^{38}$ L. Bocquet and E. Charlaix, Chem. Soc. Rev. 39, 1073 (2010).

${ }^{39}$ S. Gruener, T. Hofmann, D. Wallacher, A. Kityk, and P. Huber, Phys. Rev. E 79, 067301 (2009).

${ }^{40}$ A. Kusmin, S. Grüner, A. Henschel, O. Holderer, J. Allgaier, D. Richter, and P. Huber, J. Phys. Chem. Lett. 1, 3116 (2010).

${ }^{41}$ O. Vincent, A. Szenicer, and A. D. Stroock, Soft Matter 12, 6656 (2016).

${ }^{42}$ B.-Y. Cao, M. Yang, and G.-J. Hu, RSC Adv. 6, 7553 (2016). 